\title{
ERRATUM
}

Due to a publishing error the article "Effect of different light curing methods on mechanical and physical properties of resin-cements polymerized through ceramic discs", published at Journal of Applied Oral Science 19(4):403-12 was printed with the following errors:

\section{Page 403}

Corresponding e-mail should be read: isicekic@gazi.edu.tr

\section{Pages 405, 408 and 409}

Title "RESULTS" should be disregarded. The topics "Water solubility and sorption", "DTS" and "Statistical analysis" are part of "MATERIALS AND METHODS" section.

\section{Page 406}

The word "models" (on caption for Figure 3) should be read "modes".

\section{Page 408}

"The Vicker's hardness number (VHN) test" should be read "The Vicker's microhardness test".

\section{Page 409}

After "Statistical analysis" the following text should be considered:

\section{RESULTS}

\section{Water solubility and sorption}

The water solubility and sorption median values and $\left(25^{\text {th }}-75^{\text {th }}\right)$ percentiles are represented in Tables 3 and 4 . Comparing the median values of solubility and sorption, Kruskal Wallis test revealed statistically significant differences among resin cement and LCU groups $(p<0.05)$. The effect of the storage time factor ( $24 \mathrm{~h}$ and 7 days) on solubility and sorption behaviors was shown to be significant for the resin cements and LCUs $(p<0.05)$. Additionally, there was no significant difference between water solubility and sorption values of high-intensity and soft-start modes of the same LCU either after $24 \mathrm{~h}$ (water solubility: $\mathrm{p}=0.635$; sorption: $\mathrm{p}=0.092$ ) or 7 days (water solubility: $p=0.145$; sorption: $p=0.189$ ).

Regarding water solubility, resin cements light polymerized with PAC showed the highest water solubility values except Bifix-QM. For Bifix-QM, the highest water 
solubility values after $24 \mathrm{~h}$ and 7 days storage in water were seen when this cement was polymerized with high-intensity mode of QTH LCU (24 h: QTH (9.91), LED (5.10), PAC (7.64); 7 days: QTH (7.88), LED (2.85), PAC (5.88)) (Table 3). A ranking for water solubility of resin cements was obtained as follows: Duolink $<$ Bifix-QM $<$ Nexus $<$ RelyX Unicem<Panavia F. Moreover, according to the water solubility results obtained from resin cements polymerized with different LCUs after $24 \mathrm{~h}$ and 7 days, LCUs were ranked as follows: LED $<Q T H<P A C(p<0.05)$.

While the highest solubility value was observed after 7 days storage in distilled water with Panavia F polymerized with soft-start mode of PAC $\left(152.14 \mu \mathrm{g} / \mathrm{mm}^{3}\right)$, the lowest was observed after 7 days storage in distilled water with Duolink polymerized with high intensity mode of QTH $\left(1.27 \mu \mathrm{g} / \mathrm{mm}^{3}\right)$ (Table 3). All the tested resin cements, except Nexus, Bifix-QM and RelyX Unicem polymerized with softstart mode of PAC, and Panavia $F$ had a decrease in water solubility values with respect to water storage duration. According to the median values of the resin cements, the highest percentage of weight change in solubility values from $24 \mathrm{~h}$ to 7 days was seen in Panavia $\mathrm{F}$ (43.44 $\%, p<0.05)$.

Regarding water sorption, sorption values measured after $24 \mathrm{~h}$ and 7 days were ranked as follows: Bifix-QM<Nexus<Duolink<RelyX Unicem<Panavia F. Furthermore, LCU ranking after $24 \mathrm{~h}$ can be shown as follows: LED<QTH<PAC. However, after 7 days the sorption values of the resin cements polymerized with LED presented statistically lower sorption values than PAC and higher values than QTH $(p<0.05)$.

The highest sorption value was obtained after The highest sorption value was obtained after 7 days storage in distilled water with Panavia F polymerized with softstart mode of PAC $\left(106.24 \mu \mathrm{g} / \mathrm{mm}^{3}\right)$, the lowest was obtained after 7 days storage in distilled water with Bifix-QM polymerized with softstart mode of QTH $\left(4.59 \mu \mathrm{g} / \mathrm{mm}^{3}\right)$ (Table 4). All the tested resin cements except Nexus polymerized with soft-start mode of QTH, high intensity modes of LED and PAC; Duolink polymerized with high intensity modes of QTH and LED, RelyX polymerized with soft-start mode of QTH and Bifix-QM polymerized with soft-start and high intensity modes of QTH had an increase in water sorption values with respect to water storage duration (Table 4). Similar to the solubility test, according to the median values of the resin cements, Panavia F showed the highest percentage of weight change in sorption values from $24 \mathrm{~h}$ to 7 days $(26.54 \%$, $\mathrm{p}<0.05)$. 


\section{Diametral tensile strength}

Results of the DTS test are presented in Table 5. Resin cement and LCU had significant effects on DTS $(p<0.05)$. However, no significant differences were detected between the polymerization modes of the same LCU $(p=0.086)$. The median DTS values of Duolink, Bifix-QM and Nexus were higher than that of RelyX Unicem and Panavia $F$ and the ranking was obtained as follows: Panavia $F<$ RelyX Unicem $<$ Nexus $<$ Bifix-QM<Duolink. Moreover, resin cements polymerized with LED LCU exhibited higher DTS values than QTH and PAC LCUs. Additionally, in all groups except Bifix-QM and Duolink polymerized with high intensity mode of PAC, DTS values decreased with the use of PAC LCU (Table 5). Duolink polymerized with soft-start mode of LED LCU had the highest median DTS value (63.86 MPa), whereas Panavia F polymerized with high intensity mode of PAC had the lowest (24.28 MPa).

\section{Vicker's microhardness}

The median VHN values and $\left(25^{\text {th }}-75^{\text {th }}\right)$ percentiles for samples are shown in Table 6. Although the data showed a significant difference for the resin cement groups and LCU groups $(p<0.05)$, the differences were not significant for the polymerization mode of the same LCU $(p=0.091)$. Comparisons for VHN median values among resin cements revealed that Panavia $\mathrm{F}<$ Nexus $<$ Duolink<RelyX Unicem<Bifix-QM. Bifix-QM polymerized with soft-start mode of LED yielded a highest median VHN value (55.25) whereas Panavia F polymerized with soft-start mode of PAC LCU presented the lowest value overall (9.76). Furthermore, QTH LCU presented higher VHN values than PAC LCU and lower values than LED LCU. 